\title{
Eficacia del proceso de sucesión en empresas familiares vinculadas a la mediana minería formal peruana
}

\section{Effectiveness of Succession Process in Family Companies related to the medium Peruvian formal mining industry}

\author{
*Schwarz Díaz, M.G. ${ }^{1}$ \\ ${ }^{1}$ Universidad de Lima, Perú
}

\section{RESUMEN}

La investigación propone una medida de la eficacia de la sucesión en empresas familiares vinculadas a la minería peruana por medio de una evaluación del cambio de valor de mercado del grupo empresarial que representan. Se revisaron 12 casos de grupos empresariales y 85 reportes corporativos de empresas familiares peruanas vinculadas a la mediana minería formal durante el período 2000-2015 encontrándose hasta cuatro categorías marcadas en el cambio de valor que expresan distintas condiciones de contexto en el cambio de enfoque y dirección de la empresa ante la sucesión en el mando de la misma. Los resultados muestran tasas de cambio en el valor de mercado $(>10 \%)$ para los casos donde el cambio se generó como consecuencia del fallecimiento inesperado del patriarca y a la vez la segunda generación estaba profesionalmente preparada para hacerse cargo de la dirección de la compañía, tasas de cambio en el rango de $<5 \%-10 \%>$ para los casos donde la segunda generación no había seguido un proceso de preparación profesional planificada para la sucesión y tasas de cambio inferiores al 5\% para los casos donde la sucesión corresponde a la coexistencia en la gestión de las distintas actividades de la empresa entre patriarca y los sucesor(es).

Palabras clave: Eficacia; sucesión; empresa familiar; competent person report; valor de mercado.

ABSTRACT
Research proposes an effectiveness measurement about succession towards
the medium Peruvian mining companies through an evaluation of the market
value change of the company group represented. Twelve company groups cases
were reviewed and 85 Peruvian family corporate companies related to the
medium formal mining industry during the $2000-2015$ period, in which up to
four categories were found as part of the value change that presents different
context conditions about focus change and company direction as a result of the
succession in charge. The results show exchange rates in the market value of
(>10\%) as consequence of succession process for the family companies' cases
where the change was professionally prepared to take over the leadership of the
company; exchange rates in the range of $5 \%$ to $10 \%$ for cases where the
second generation had not followed a process of professionally planned
preparation of succession; and lower change rates of $5 \%$ for cases where the

*Autor Correspondiente: Max Guillermo Schwarz Díaz, Universidad de Lima, Lima - Perú E-mail: mschwarz@ulima.edu.pe.

Fecha de recepción: setiembre 2016; Fecha de aceptación: octubre 2016 
succession corresponds to the coexistence in the management of the various activities of the company between patriarch and successor(s).

Keywords: Effectiveness; succession; family company; competent person report; market value.

\section{INTRODUCCIÓN}

La empresa familiar constituye el pilar fundamental de la generación de negocios en el Perú representando el $90 \%$ de las empresas formales con una contribución del 75\% del PBI peruano (PwCReport, 2013) sin embargo la mayoría lamentablemente desaparece con el emprendedor, fundador original o patriarca, producto de la ausencia procesos de sucesión organizados que permitan su supervivencia por múltiples razones que determinan que solo el $15 \%$ de las empresas familiares en el Perú sobrevive a la tercera generación. (Report STEP, 2014). En este contexto la empresa familiar en el Perú es la forma predominante de organización empresarial y representa el

principal soporte de la economía y la primera fuente de generación de empleo nacional en una economía creciente con un grado de informalidad cercano al 80\% (MEF-Perú 2015, WDI 2014).

El proceso de sucesión es el conjunto de actividades interrelacionadas que permiten producir un cambio de mando en la organización empresarial en determinados contextos y bajo condiciones especificadas por una combinación entre la naturaleza del negocio y la naturaleza de la organización que lo administra. Se trata de un proceso complejo donde interviene factores psicológicos, culturales, económico-financieros, legales-regulatorios, tecnológicos, comerciales y administrativos vinculados a la familia propietaria y a la organización que ha desarrollado.

Los procesos de sucesión en empresas familiares se encuentran fuertemente influenciados por variables como la personalidad del patriarca, la cultura familiar, la composición accionaria de la compañía, el grado de preparación profesional de los potenciales sucesores, la existencia de intereses familiares y personales de personas vinculadas al entorno familiar y empresarial, los compromisos y acreencias adquiridas, la naturaleza de los contratos, la posición de dominio sobre la generación de caja, los conflictos de intereses del entorno, el grado de control gerencial, la dependencia en la toma de decisiones, el contexto legal, la existencia de herederos, el tamaño y la complejidad de la empresa entre otros que afectan la eficacia del proceso y su impacto en el cambio de valor de las compañías.

En el Perú las principales empresas familiares vinculadas a la mediana minería formal corresponden a familias tradicionalmente mineras vinculadas a un patriarca emprendedor original que desarrolla su modelo de negocio inicialmente como explorador, contratista y pequeño productor hasta convertirlo en una organización minera con operaciones de extracción, beneficio y comercialización de metales. El patriarca emprendedor generalmente se caracteriza por una concentración masiva de propiedad, una dirección vertical y paternal de la empresa con la gestión de un entorno formado por personal de extrema confianza y un proceso sostenido de reinversión de utilidades en sus propias operaciones o el financiamiento de sus nuevos emprendimientos con el flujo de caja de sus minas en operación para un crecimiento organizado y sostenido.

La literatura vinculada a empresas familiares, su cultura y su comportamiento organizacional han sido materia de estudio de diferentes investigaciones 
cualitativas y cuantitativas como puede apreciarse en los trabajos de Botero, I. C. et al (2015), Cooper, J. T., Kidwell, R. E., \&Eddleston, K. A. (2013), Diéguezsoto, J., López-delgado, P., \& Rojo-Ramírez, A. (2015)., Rothwell, William J. (2016), Xi, J., Kraus, S., Filser, M., \& Kellermanns, F. W. (2015) y Zou, W., Chiu, Y., \&Hsu, C. (2014), quienes han realizado investigaciones enfocadas a comprender la naturaleza compleja de la empresa familiar, su importancia y su participación como contribución a la sociedad y al mercado.

El problema de la eficacia del proceso de sucesión en empresas familiares ha sido ampliamente tratado en la literatura por diversos autores como puede apreciarse en los trabajos recientes de Bjuggren, P., \&Sund, L. (2014), Boyd, B.et al (2014), Carey et al (2000), Koffi, V. et al (2014), Koropp, C., Grichnik, D., \&Gygax, A. F. (2013). ySimões Vieira, E. F. (2014), quienes han desarrollado aspectos específicos vinculados a los factores que afectan el proceso de sucesión en empresas familiares en distintos contextos y con diferente grado de complejidad.

El problema de la valorización de empresas ha cambiado de giro y las empresas valen más por su capacidad de generación de caja que por sus activos, es decir una empresa es más atractiva cuando tiene una capacidad de generación de caja creciente y no tanto por el tamaño de sus activos lo cual implica que la capacidad de generar caja depende del modelo de negocio tanto como de la capacidad de la administración para mantener creciente esa generación de caja. El modelo refleja el impacto del capital intelectual y relacional basado en el recurso humano, su competencia y calificaciones para agregar valor en del mercado.

\section{METODOLOGÍA}

La investigación es descriptiva, explicativa y relacional planteada sobre la base de la estadística comercial de una muestra no aleatoria de tipo intencional configurada por 12 grupos económicos y 85 reportes corporativos de empresas familiares peruanas vinculadas a la mediana minería formal peruana obtenidos entre los años 2000 al 2015. Se ha recolectado información bursátil, reportes bancarios y análisis de empresas de la Bolsa de Valores de Lima (BVL), Toronto Stock Exchange (TSE), London Stock Exchange (LSE) y la Superintendencia de Mercado de Valores (SMV-Perú) para establecer el valor de mercado de las compañías estudiadas a partir de su generación de caja, el valor de los activos, el nivel de endeudamiento y capacidad de la gerencia para sobrevivir en el mercado medida con el instrumento normalizado en escala (0-10) para Competence Person Report (CPR) aplicado a la data de estudio.

La investigación se concentra en el cambio de valor de las empresas antes y después del proceso de sucesión para lo cual hace uso del proceso de valorización de empresas mineras basado en la siguiente ecuación de referencia:

$V M=$ Valor de activos $+(n) *($ EBITDA $)-$ Valor de deuda

Donde " $n$ " se calcula a partir del Competent Person Report (CPR) que en el caso de la investigación se ha aplicado a los 12 grupos empresariales materia de estudio y representa el tiempo en años que la evaluación determina para la capacidad de la gerencia de gestionar la reposición de reservas, la retención de talento y hacer crecer una sostenida generación de caja para penetrar y crecer en el mercado. Ref.: Ross et al (2012), Stowe J.D. (2002), Financial Due Diligence Reports for IPO in TSE/LSE/BVL (2015). 


\section{RESULTADOS}

El valor de mercado de las empresas familiares peruanas como grupos económicos empresariales representativos vinculados a la mediana minería formal es de $\$ 18,692$ MM (la valorización únicamente contempla empresas mineras, excluyendo sus negocios complementarios, corporativos o vinculados a otros sectores empresariales). La distribución de las valorizaciones puede revisarse en el Tabla 1.1 donde podemos apreciar el valor de los activos, la capacidad de generación de caja (medida como EBITDA), el intangible intelectual y relacional expresado a partir del instrumento CPR, el componente de deuda y el valor de mercado de las empresas materia de estudio calculado a partir de la ecuación 1.

Tabla 1.1.

Valor de Mercado de Empresas familiares vinculadas a la mediana minería formal peruana

\begin{tabular}{|c|c|c|c|c|c|c|c|}
\hline \multirow[b]{2}{*}{ Grupo Económico } & \multirow[b]{2}{*}{ Familia } & \multicolumn{6}{|c|}{ Estado de la empresa familiar como grupo económico al 2015} \\
\hline & & $\begin{array}{l}\text { Activos } \\
\text { (\$MM) }\end{array}$ & $\begin{array}{l}\text { EBITDA } \\
\text { (\$MM) }\end{array}$ & CPR: $\mathrm{n}$ & Deuda/Act. & Bolsa & $\begin{array}{c}\text { Valor de } \\
\text { Mercado }\left(^{*}\right)\end{array}$ \\
\hline Buenaventura & Benavides & $\$ 1,126$ & $\$ 105$ & 16 & 0.1633 & BVL/NYSE & $\$ 2,622$ \\
\hline Grupo Breca & Brescia-Cafferata & $\$ 2,613$ & $\$ 149$ & 10 & 0.1862 & BVL/NYSE & $\$ 3,616$ \\
\hline Volcan & Letts Colmerares & $\$ 728$ & $\$ 226$ & 10 & 0.4496 & BVL & $\$ 2,661$ \\
\hline Hochschild & Hochschild & $\$ 737$ & $\$ 133$ & 18 & 0.1845 & LSE & $\$ 2,995$ \\
\hline Aruntani-Arasi & Del Castillo & $\$ 213$ & $\$ 68$ & 8 & 0.1224 & No Lista & $\$ 731$ \\
\hline Consorcio Minero Horizonte & Navarro Grau & $\$ 378$ & $\$ 94$ & 8 & 0.3412 & No Lista & $\$ 1,001$ \\
\hline MARSA & Marsano & $\$ 325$ & $\$ 87$ & 10 & 0.3112 & No Lista & $\$ 1,094$ \\
\hline PODEROSA & Arias Dávila & $\$ 267$ & $\$ 82$ & 10 & 0.2456 & No Lista & $\$ 1,021$ \\
\hline COMARSA-LA VIRGEN & Sanchez Paredes & $\$ 312$ & $\$ 102$ & 8 & 0.3542 & No Lista & $\$ 1,017$ \\
\hline Gubbins & Gubbins & $\$ 215$ & $\$ 53$ & 8 & 0.3374 & No Lista & $\$ 566$ \\
\hline Fortuna & Ganoza & $\$ 425$ & $\$ 68$ & 8 & 0.1383 & BVL/TSE & $\$ 910$ \\
\hline Caudalosa & Raffo & $\$ 187$ & $\$ 42$ & 8 & 0.3556 & No Lista & $\$ 457$ \\
\hline \multicolumn{8}{|c|}{$\left({ }^{*}\right)$ Valor de Mercado $=$ Activos $+n($ EBITDA $)-$ Deudas } \\
\hline \multicolumn{8}{|c|}{ Nota: La valorización solo contempla inversiones y activos (exclusivamente minas) en el Perú } \\
\hline
\end{tabular}

Fuente: Elaboración propia extraida de LSE, NYSE, BVL y SMV-Perú Período 2000-2015

La tasa de cambio en el valor de mercado antes y después de la sucesión puede apreciarse en la Tabla 1.2 considerándose un periodo de 2 años a partir de la fecha de sucesión en cada caso con el objeto de poder verificar el cambio en los resultados como consecuencia de la nueva gestión empresarial que asume el mando ante el retiro de los patriarcas o fundadores de la organización.

Tabla 1.2

Tasa de Cambio en el valor de mercado antes y despues del proceso de sucesión

\begin{tabular}{|c|c|c|c|c|c|c|c|c|c|c|c|}
\hline \multirow[b]{2}{*}{ Grupo Económico } & \multirow[b]{2}{*}{ Familia } & \multirow[b]{2}{*}{$\begin{array}{c}\text { Año } \\
\text { Sucesión }\end{array}$} & \multicolumn{3}{|c|}{ Estado Antes de la sucesión } & \multicolumn{3}{|c|}{ Estado después de la sucesión } & \multicolumn{2}{|c|}{ Cambio de Valor } & \multirow[b]{2}{*}{ Periodo Análisis } \\
\hline & & & $\begin{array}{l}\text { Valor de } \\
\text { Mercado }\end{array}$ & $\begin{array}{l}\text { Valor de } \\
\text { Acción }\end{array}$ & Beta & $\begin{array}{l}\text { Valor de } \\
\text { Mercado }\end{array}$ & $\begin{array}{l}\text { Valor de } \\
\text { Acción }\end{array}$ & Beta & $\begin{array}{c}\text { Var \% Valor } \\
\text { Acción }\end{array}$ & $\begin{array}{c}\text { Var \% Valor } \\
\text { Mercado }\end{array}$ & \\
\hline \begin{tabular}{|l|} 
Buenaventura \\
\end{tabular} & Benavides & 2014 & $\$ 2,318$ & 11.62 & 0.92 & $\$ 2,622$ & 12.73 & 0.93 & $9.55 \%$ & $13.12 \%$ & Jul 2014-Jul2016 \\
\hline Grupo Breca & Brescia-Cafferata & 2013 & $\$ 3,100$ & 1.18 & 0.82 & $\$ 3,600$ & 1.33 & 0.87 & $12.71 \%$ & $16.13 \%$ & Jul 2013-Jul 2015 \\
\hline Volcan & Letts Colmerares & 2010 & $\$ 2,250$ & 3.12 & 1.93 & $\$ 2,400$ & 3.35 & 1.82 & $7.69 \%$ & $6.67 \%$ & Jul 2010 - Jul 2012 \\
\hline Hochschild & Hochschild & 1998 & $\$ 675$ & No definido & No definido & $\$ 750$ & No definido & No definido & - & $11.11 \%$ & Jul 1998 - Jul 2000 \\
\hline Aruntani-Arasi & Del Castillo & En proceso & \multicolumn{9}{|c|}{ No definido } \\
\hline \begin{tabular}{|l|} 
Consorcio Minero Horizonte \\
\end{tabular} & Navarro Grau & En proceso & \multicolumn{9}{|c|}{ No definido } \\
\hline MARSA & Marsano & 1999 & $\$ 783$ & No definido & \begin{tabular}{|l|} 
No definido \\
\end{tabular} & $\$ 925$ & \begin{tabular}{|l|} 
No definido \\
\end{tabular} & \begin{tabular}{|l|} 
No definido \\
\end{tabular} & - & $18.14 \%$ & \begin{tabular}{|l|} 
Jul 1999-Jul 2001 \\
\end{tabular} \\
\hline \begin{tabular}{|l} 
PODEROSA \\
\end{tabular} & Arias Dávila & 2011 & $\$ 683$ & \begin{tabular}{|l|} 
No definido \\
\end{tabular} & \begin{tabular}{|l|} 
No definido \\
\end{tabular} & $\$ 897$ & \begin{tabular}{|l|} 
No definido \\
\end{tabular} & No definido & - & $31.33 \%$ & \begin{tabular}{|l|} 
Jul 2011-Jul 2013 \\
\end{tabular} \\
\hline COMARSA-LA VIRGEN & Sanchez Paredes & En proceso & \multicolumn{9}{|c|}{ No definido } \\
\hline Gubbins & Gubbins & 2001 & $\$ 450$ & & No definido & $\$ 472$ & & No definido & - & $4.89 \%$ & Jul 2001 - Jul 2003 \\
\hline Fortuna & Ganoza & No inicia & \multicolumn{9}{|c|}{ No definido } \\
\hline Caudalosa & Raffo & 2010 & $\$ 380$ & No definido & No definido & $\$ 397$ & No definido & No definido & - & $4.47 \%$ & Jul 2010 - Jul 2012 \\
\hline
\end{tabular}


La relación entre el cambio de valor y el contexto de la sucesión en el cambio de mando de las empresas familiares se encuentra correlacionada con la calificación del contexto en el rango teórico esperado. Los resultados pueden revisarse en la Tabla 1.3 adjunta.

Tabla 1.3

Relación entre el cambio de valor y el contexto de la sucesión

\begin{tabular}{|l|c|c|c|c|c|c|}
\hline Grupo Económico & Familia & $\begin{array}{c}\text { Año } \\
\text { Sucesión }\end{array}$ & Contexto & $\begin{array}{c}\text { Cambio de } \\
\text { Valor }\end{array}$ & $\begin{array}{c}\text { Rango } \\
\text { Teórico }\end{array}$ & Correlación \\
\hline & & & & & & \\
\hline Buenaventura & Benavides & 2014 & $\mathrm{~A}$ & $13.12 \%$ & $>10 \%$ & $\mathrm{r} 2=0.83$ \\
\hline Grupo Breca & Brescia-Cafferata & 2013 & $\mathrm{~A}$ & $16.13 \%$ & $>10 \%$ & $\mathrm{r} 2=0.88$ \\
\hline Volcan & Letts Colmerares & 2010 & $\mathrm{~B}$ & $6.67 \%$ & $>5 \%$ & $\mathrm{r} 2=0.80$ \\
\hline Hochschild & Hochschild & 1998 & $\mathrm{~A}$ & $11.11 \%$ & $>10 \%$ & $\mathrm{r} 2=0.89$ \\
\hline Aruntani-Arasi & Del Castillo & En proceso & \multicolumn{5}{|c|}{ No definido } \\
\hline Consorcio Minero Horizonte & Navarro Grau & En proceso & \multicolumn{5}{|c|}{ Nofinido } \\
\hline MARSA & Marsano & 1999 & B & $18.14 \%$ & $>5 \%$ & $\mathrm{r} 2=0.98$ \\
\hline PODEROSA & Arias Dávila & 2011 & $\mathrm{~A}$ & $31.33 \%$ & $>10 \%$ & $\mathrm{r} 2=0.93$ \\
\hline COMARSA-LA VIRGEN & Sanchez Paredes & En proceso & \multicolumn{5}{|c|}{ No definido } \\
\hline Gubbins & Gubbins & 2001 & C-D & $4.89 \%$ & $<5 \%$ & $\mathrm{r} 2=0.34$ \\
\hline Fortuna & Ganoza & No inicia & \multicolumn{5}{|c|}{ No definido } \\
\hline Caudalosa & Raffo & 2010 & C-D & $4.47 \%$ & $<5 \%$ & $\mathrm{r} 2=0.88$ \\
\hline
\end{tabular}

\begin{tabular}{|l|c|}
\hline Condiciones de Contexto de la sucesión & $\%$ Casos \\
\hline A: Fallecimiento inesperado de patriarca con preparación profesional planeada de sucesores & $50 \%$ \\
\hline B: Fallecimiento inesperado de patriarca sin preparación profesional planeada de sucesores & $25 \%$ \\
\hline C: Gobierno gerencial compartido hasta el cambio de mando empresarial por retiro o fallecimiento & \multirow{2}{*}{$25 \%$} \\
\hline D: Gobierno gerencial dominante hasta el cambio gerencial manteniendo algun grado de control & \\
\hline
\end{tabular}

Fuente: Elaboración propia extraida de LSE, NYSE, BVL y SMV-Perú Período 2000-2015

\section{DISCUSIÓN}

Los resultados muestran el cambio en el valor de mercado de la compañía como consecuencia de la sucesión determinando características particulares de los casos estudiados. De esta manera podemos verificar la existencia de hasta 4 tipos de casos claramente definidos. El primer caso (A) (50\% de las empresas que llegaron a completar la sucesión) corresponde a empresas familiares donde el cambio se generó como consecuencia del fallecimiento inesperado del patriarca y a la vez la segunda generación estaba profesionalmente preparada para hacerse cargo de la dirección de la compañía. En estos casos se muestran tasas de cambio en el valor de mercado ( $>10 \%$ ) a partir del segundo año del proceso de sucesión con una correlación superior a 0.8 (Rango r2 $=0.83-0.93$ )

El segundo caso (B) ( $25 \%$ de las empresas que llegaron a completar la sucesión) corresponde a empresas familiares donde el cambio se generó como consecuencia del fallecimiento inesperado del patriarca y la segunda generación no había seguido un proceso de preparación profesional planificada para la sucesión. En estos casos se muestra una tasa media de cambio en el valor de mercado $<5 \%-10 \%>$ a partir del segundo año del proceso de sucesión con una correlación superior a 0.4 (Rango r2=0.47-0.90)

El tercer (C) y cuarto caso (D) ( $25 \%$ de las empresas que llegaron a completar la sucesión) corresponde a empresas familiares donde el cambio se generó como consecuencia de un largo proceso de gerencia compartida (C) donde el patriarca y su sucesor(es) coexisten en la gestión de las distintas actividades de la empresa hasta el retiro del patriarca o bien a casos (D) que se 
generan como consecuencia de un proceso de gerencia compartida donde el patriarca y su sucesor(es) coexisten en la gestión de las distintas actividades de la empresa, pero el patriarca se resiste a retirarse o mantiene algún grado de control real, aunque no formal en la empresa. En estos casos, luego de cambio generacional formal se muestra una tasa media de tasa de cambio en el valor de mercado inferior al $5 \%$ a partir del segundo año del proceso de sucesión con una correlación superior a 0.4 (Rango r2=0.47-0.90)

\section{CONCLUSIONES Y RECOMENDACIONES}

La investigación demuestra que, si bien el proceso de sucesión de mando en las empresas familiares vinculadas a la mediana minería formal no es homogéneo, los resultados reflejan un mayor incremento de valor durante los dos primeros años a partir de la fecha de sucesión en los casos donde los sucesores tenían una mayor preparación para asumir la dirección de la organización como consecuencia de un proceso planeado que se acelera con el fallecimiento del patriarca, mostrando a la vez que los casos de menor incremento de valor se dan en el caso de sucesiones no planificadas donde el patriarca se resiste a dejar la dirección de la empresa, se generan conflictos internos o existe un gobierno compartido en la dirección de la organización. El incremento de valor en el $100 \%$ de los casos estudiados puede explicarse a partir de la introducción de nuevos estilos de gerencia en la dirección y modernización ejercida por los sucesores en un contexto de economía creciente como la peruana en el mismo período de análisis siendo la diferencia atribuible a la naturaleza de la sucesión materia de estudio. La investigación determina un incremento de valor en todos los casos que además en la mayoría de ellos se vuelve sostenible puesto que las empresas con procesos de sucesión más estables han logrado al 2015 un proceso de internacionalización y la conversión publica por medio del listado en bolsas de valores locales e internacionales con un consecuente incremento adicional de valor que no hubiera sido posible o tendría una menor probabilidad de ocurrencia en el contexto de la administración de los patriarcas o fundadores originales de las compañías materia de estudio. Los casos estudiados permiten concluir que la eficacia del proceso de sucesión en términos de cambio de valor de la empresa depende en mayor medida del grado de preparación de los sucesores para asumir la dirección de la organización acercándose a los niveles categorizados A y B.

\section{REFERENCIAS BIBLIOGRAFÍCAS}

Bjuggren, P., \& Sund, L. (2014). A contractual perspective on succession in family firms: A stakeholder view. European Journal of Law and Economics, 38(2), 211-225. doi: http://dx.doi.org/10.1007/s10657-012-9331-6.

Botero, I. C., Gomez Betancourt, G., Betancourt Ramirez, J. B., \& Lopez Vergara, M. P. (2015). Family protocols as governance tools. Journal of Family Business Management, 5(2), 218.

Boyd, B., Botero, I. C., \& Fediuk, T. A. (2014). Incumbent decisions about succession transitions in family firms: A conceptual model. International Journal of Financial Studies, 2(4), 335-358. doi:http://dx.doi.org/10.3390/ijfs2040335

Carey, Dennis C., Ogden, Dayton, and Roland, Judith A. CEO Succession: Lessons from the Trenches. Cary, US: Oxford University Press (US), 2000. ProQuest ebrary.Web. 17 August 2016.

Cooper, J. T., Kidwell, R. E., \&Eddleston, K. A. (2013).Boss and parent, employee and child: Work-family roles and deviant behavior in the family firm. Family Relations,62(3), 457-471. 
Danes, S. M., Stafford, K., \&JohnbenTeik-Cheok Loy.(2007). Family business performance: The effects of gender and management*. Journal of Business Research, 60(10), 1058.

Diéguez-soto, J., López-delgado, P., \&Rojo-ramírez, A. (2015). Identifying and classifying family businesses. Review of Managerial Science, 9(3), 603-634. doi:http://dx.doi.org/10.1007/s11846-014-0128-6

Duh, M. (2014). Family business succession as knowledge creation process. Kybernetes, 43(5), 699-714. doi:http://dx.doi.org/10.1108/K-08-2013-0172

Koffi, V., Fillion, G., Ekionea, J. B., \& Morris, T. (2014).Family business succession: what are the ways used by the men business managers to legitimize their successors? The Entrepreneurial Executive, 19, 111-129.

Koropp, C., Grichnik, D., \&Gygax, A. F. (2013).Succession financing in family firms.Small Business Economics, 41(2), 315-334. doi: http://dx.doi.org/10.1007/s11187-012$9442-z$

Memoria Anual - Hochschild Mining PLC Ed. 2008, 2009, 2010, 2011, 2012, 2013, 2014 y 2015.

Memoria Anual - Compañía de Minas Buenaventura Ed. 2013, 2014 y 2015.

Memoria Anual - Volcan Cía. Minera S.A.A. Ed. 2010, 2011, 2012, 2013, 2014 y 2015.

Rothwell, William J. Effective Succession Planning: Ensuring Leadership Continuity and Building Talent from Within (3). Saranac Lake, US: AMACOM, 2005. ProQuest ebrary.Web. 17 August 2016.

Olson, P. D., Zuiker, V. S., Danes, S. M., Stafford, K., Heck, R. K., \& Duncan, K. A. (2003). The impact of the family and the business on family business sustainability. Journal Of Business Venturing, 18(5), 639. doi:10.1016/S0883-9026(03)00014-4

Price Waterhouse Report PwC-Perú 2013

Ross, S. A., Westerfield, R. W., Jaffe, J. F., Carril, V. P., \&Gómez, M. A. J. (2012). Finanzas corporativas. México: MacGraw-Hill.

Sanborn, C., \& Paredes, Á. (2014). Grupos económicos y bonanza minera en el perú. el caso de cinco grupos mineros nacionales. Apuntes, 41(74), 235-237.

Simões Vieira, E.,F. (2014). The effect on the performance of listed family and non-family firms. Managerial Finance, 40(3), 234-253. doi:http://dx.doi.org/10.1108/MF-062013-0134

Stowe J.D.,T.R. Robinson, J.E.Pinto y D.W. McLeavey (2002) Analysis of equity investments: Valuation. AIMR (Association for Investment Management and Research).

Xi, J., Kraus, S., Filser, M., \&Kellermanns, F. W. (2015).Mapping the field of family business research: Past trends and future directions. International Entrepreneurship and Management Journal, 11(1), 113-132. doi:http://dx.doi.org/10.1007/s11365-0130286-z

Zou, W., Chiu, Y., \& Hsu, C. (2014).Performance appraisals between family businesses and non-family businesses. International Journal of OrganizationalInnovation(Online), $7(1), 36-45$. 